'Servicio de Oftalmología Hospital Dr. Sótero del Río, Servicio de Salud Metropolitano Sur Oriente. Santiago, Chile.

${ }^{2}$ Centro de Epidemiología y Políticas de Salud Pública, Facultad de Medicina, Clínica Alemana, Universidad del Desarrollo. Santiago, Chile. ${ }^{\mathrm{a}} \mathrm{PhD}$.

Declaración de que no hubo fuente de apoyo financiero.

Recibido el 22 de agosto de 2016, aceptado el 18 de mayo de 2017.

Correspondencia a: Dra. Trinidad Covarrubias Servicio de Oftalmología Hospital Dr. Sótero del Río, Servicio de Salud Metropolitano Sur Oriente. Santiago, Chile. tcovarrubiasc@gmail.com

\section{Tamizaje en el diagnóstico y prevalencia de retinopatía diabética en atención primaria}

\author{
TRINIDAD COVARRUBIAS ${ }^{1}$, IRIS DELGADO ${ }^{2, a}$, \\ DANIEL ROJAS ${ }^{1}$, MARCELO CORIA ${ }^{1}$
}

Background: Diabetic retinopathy is the first cause of blindness during working years. Aim: Provide knowledge of screening coverage, prevalence and level of diabetic retinopathy in patients that belong to the Cardiovascular Health Program in primary care. Material and Methods: Analysis of retinographies performed to 9076 diabetic patients aged $61 \pm 13$ years (61\% women) adscribed to a Cardiovascular Health program in primary care centers of South-East Metropolitan Santiago. The examination was carried out by the evaluation of retinographies by trained optometrists. Results: The coverage of the screening program was $21 \%$. The prevalence of sight threatening diabetic retinopathy was $3,1 \%$. The prevalence of these entities was $45 \%$ higher in people aged between 18 and 44 years than in older people. Their prevalence in urban communities was $32 \%$ higher than in rural locations. Conclusions: The coverage of the screening program is low. Diabetic patients aged 18 to 44 years and those coming from urban communities have a higher prevalence of severe non-proliferative and proliferative diabetic retinopathy.

(Rev Med Chile 2017; 145: 564-571)

Key words: Diabetic Retinopathy; Diagnosis; Delivery of Healthcare; Mass Screening; Prevalence of Healthcare.

\section{L} a retinopatía diabética (RD) es considerada en los países desarrollados como la primera causa de ceguera en personas en edad laboral. Según Furtado et $\mathrm{al}^{1}$, la RD es la segunda causa de ceguera en Latinoamérica, después de la catarata. Por otro lado, la diabetes mellitus (DM) es actualmente uno de los problemas más serios que enfrenta la salud pública mundial, estimándose que en el año 2000 existían en el planeta alrededor de 170 millones de personas afectadas. Esto conlleva a que la proyección al año 2030 sea de 360 millones de personas ${ }^{2}$. En Chile, según la Encuesta Nacional de Salud 2009-2010, la prevalencia estimada de DM en mayores de 15 años es de 9,4\%, siendo ésta superior en los mayores de 65 años y en aquellos con bajo nivel educacional ${ }^{3}$. Así mismo, la cobertura efectiva de la diabetes -estimada según valores de hemoglobina glicosilada menores a 7\%- es baja, apreciándose una prevalencia nacional de cumplimiento de la meta de tan sólo 34,3\% (IC 95\%: 27,6-41,7). Además, sólo 7,6\% de los diabéticos reporta conocer su condición de portador de $\mathrm{RD}^{4}$.

Se considera que la RD es la complicación más frecuente de la DM. Estudios internacionales han demostrado que la prevalencia de la complicación está directamente relacionada con el tiempo de evolución y el control metabólico de dicha enfer- 
medad $^{5}$. En 2012, un meta análisis que incluyó a 12.620 diabéticos, mostró una prevalencia de $\mathrm{RD}$ general de $35,4 \%$, teniendo $11,7 \%$ de éstos algún grado de RD que amenaza la visión (RD proliferativa o edema macular $)^{6}$. Cifras Latinoamericanas estiman que la prevalencia de RD es de 20 a $30 \%$ entre los pacientes con DM y 5\% de ellos presenta alto riesgo de ceguera ${ }^{7}$. No obstante, en Chile resultan escasas las publicaciones al respecto. Un estudio realizado por Verdaguer et al, arrojó ciertas luces en relación a un programa de tamizaje masivo llamado "Día D", el cual incluyó a 1.348 pacientes, siendo hasta el momento la investigación de referencia en el país. Posteriormente, otra investigación llevada a cabo por Abuabuad et al en 2014, evaluó a 468 pacientes. La prevalencia de RD en estos estudios fue de $28,5 \%$ y $24,8 \%$ respectivamente $e^{8,9}$.

La detección precoz de la RD resulta fundamental para evitar la ceguera. La Asociación Americana de Diabetes y la Academia Americana de Oftalmología afirman que, si se siguen adecuadamente las pautas de tamizaje y se trata oportunamente a los pacientes, la reducción del riesgo de ceguera se sitúa entre $12 \%$ y $28 \%{ }^{10-12}$. Esta patología resulta ser un problema importante de salud pública (que se proyecta estable o en creciente prevalencia) que cumple los criterios éticos y técnicos exigidos por la OMS para implementar programas de tamizaje. El tamizaje disminuye los costos del tratamiento, ya que la RD que amenaza la visión tiene síntomas tempranos que pueden ser detectados, pudiendo incluso ser prevenida o disminuida la velocidad de su progresión. Adicionalmente, se dispone de la panfotocoagulación, un tratamiento efectivo demostrado ${ }^{13-16}$. Para los diabéticos tipo 2, el protocolo actual de tamizaje en Chile es la realización de un control de fondo de ojo por oftalmólogo que, en caso de no presentar patología, se realizaría anualmente ${ }^{17}$.

El año 2004, el Servicio de Salud Metropolitano Sur Oriente (SSMSO) implementó un sistema de tamizaje, vía retinografía digital, instalado en Unidades de Atención Primaria Oftalmológicas (UAPO), en el marco de los pacientes adheridos al Programa de Salud Cardiovascular (PSCV) ${ }^{18}$. De un total de 1.110 .988 personas inscritas en establecimientos del nivel primario de salud (APS), $79,4 \%$ (881.476) está adscrito al PSCV del SSMSO. De ellos, 4,5\% (43.588) tiene diagnóstico de DM (incluyendo tipo 1 y 2 , y excluyendo a los diabé- ticos de la comuna de La Florida) ${ }^{19}$. Actualmente no hay estudios que evalúen la efectividad del tamizaje de RD en APS realizado por tecnólogos médicos oftalmológicos (TM). El objetivo de este estudio es analizar la cobertura del tamizaje en los pacientes adscritos al PSCV, la prevalencia y grado de severidad de la RD en SSMSO y en las comunas del servicio, con el fin de obtener información que permita mejorar dicho tamizaje.

\section{Material y Métodos}

Estudio retrospectivo analítico de pacientes diabéticos adheridos al PSCV de la atención primaria del SSMSO entre enero de 2014 y junio de 2016.

La población de estudio corresponde al universo de personas evaluadas en el período mencionado, incluyendo población urbana y rural beneficiaria del SSMSO. En un total de 9.222 pacientes, se realizaron 11.426 retinografías, lo que da un promedio de 1,24 evaluaciones por persona. Para efectos de este estudio se seleccionó sólo la primera retinografía. Del total de éstas, se excluyeron $612(6,7 \%)$ dado que el resultado fue dudoso (fotografía borrosa no concluyente). Dichos pacientes fueron derivados para evaluación por oftalmólogo en el Centro de Diagnóstico y Terapéutico (CDT) del Complejo Asistencial Dr. Sótero del Río (CASR) y no fueron considerados en el presente estudio. También se excluyó a las personas menores de 18 años por dudas en la consignación de su edad y a los pacientes de la comuna de La Florida, cuyo programa de tamizaje es ejecutado en el Hospital de la Florida.

El tamizaje se realizó mediante retinógrafo digital Polaroid $45^{\circ}$ (Canon CR2). Estudios internacionales reportan con el uso de esta técnica, sensibilidad fluctuante entre 88 y $93 \%$, y especificidad de $87 \%$, con una tasa de fallo técnico (retinografías no evaluables) menor a 5\% ${ }^{20-22}$. Los pacientes de las comunas de Puente Alto, San Ramón y La Pintana fueron evaluados en las UAPO de dichas comunas. En el caso de los inscritos en centros APS de Pirque, La Granja, San José de Maipo y en la red Áncora de la Pontificia Universidad Católica, las retinografías fueron realizadas en el CDT del CASR.

Los estudios fueron efectuados por TM, previamente capacitados por profesionales de oftal- 
Tabla 1. Escala de Severidad de la Retinopatía Diabética

\begin{tabular}{|ll|}
\hline $\begin{array}{l}\text { Nivel de severidad propuesto } \\
\text { para la RD }\end{array}$ & Hallazgos observados en la oftalmoscopía dilatada \\
\hline No hay retinopatía aparente & Sin anormalidades \\
\hline RDNP Leve & Sólo microaneurismas \\
\hline RDNP Moderada & Más que sólo microaneurismas pero menos que RDNP Severa \\
\hline RDNP Severa & $\begin{array}{l}\text { Alguno de: más de } 20 \text { hemorragias retinales en alguno de los } 4 \text { cuadrantes, } \\
\text { rosarios venosos en } 2 \text { o cuadrantes y/o anomalías microvasculares intraretinales } \\
\text { prominentes en } 1 \text { cuadrante, asociado a la ausencia de signos de RDP }\end{array}$ \\
\hline RDP & 1 o más de los siguientes criterios: neovascularización, hemorragia preretinal o vítrea \\
\hline
\end{tabular}

RD: retinopatía diabética. RDNP: retinopatía diabética no proliferativa. RDP: Retinopatía diabética proliferativa. Fuente: Ref 17 (traducida).

mología del CASR. Se obtuvieron imágenes de $45^{\circ}$, previa dilatación pupilar con tropicamida $1 \%$, las cuales fueron clasificadas por el TM siguiendo la tabla adaptada ${ }^{23}$ (Tabla 1$)$.

Los resultados de todos los pacientes evaluados fueron consignados en una plataforma online, en la página web del SSMSO llamada "fondo de ojo", resguardando los datos sensibles de las personas. Los pacientes con retinopatía diabética no proliferativa (RDNP), moderada y severa, retinopatía diabética proliferativa (RDP) y con sospecha de Edema Macular Diabético (EMD) fueron derivados al Servicio de Oftalmología del CASR para estudio y manejo de la retinopatía. La información fue proporcionada por la unidad de estudios de la subdirección de gestión asistencial del SSMSO bajo completa anonimidad. Asimismo, el comité de ética de dicho servicio aprobó la presente investigación.

Se analizaron las diferencias en la gravedad de RD según variables demográficas: sexo, edad, comuna y condición urbano/rural de la comuna. El análisis estadístico incluyó descripción de frecuencias absolutas y relativas en las variables categóricas y estadísticas de tendencia central para la variable edad. La cobertura del tamizaje se estimó utilizando como numerador la cantidad de retinografías y como denominador, el número de diabéticos del PSCV. Del mismo modo, se realizó un análisis bivariado entre la severidad de la RD y las variables demográficas. Se consideró diferencia estadística para $\mathrm{p}<0,05$. Todos los análisis se llevaron a cabo en SPSS versión 21.0 (Illinois, USA).

\section{Resultados}

De un total de 9.076 pacientes tamizados, considerando la primera retinografía realizada, la Tabla 2 muestra las características demográficas de la población en estudio. De esta manera, se observa que 6 de cada 10 pacientes son mujeres, aproximadamente $90 \%$ son mayores de 45 años y el promedio de edad es de 61 años (DS $\pm 13,4$ años), con una diferencia de 3 años más para las personas que residen en áreas urbanas $(\mathrm{p}<$ $0,001)$.

Tabla 2. Características demográficas de la población de estudio

\begin{tabular}{|llrr|}
\hline Variables & & $\mathbf{n}$ & $\mathbf{9}$ \\
& & $\mathbf{9 . 0 7 6}$ & $\mathbf{1 0 0}$ \\
Sexo & Hombre & 3.492 & 38,5 \\
& Mujer & 5.584 & 61,5 \\
Comuna & Puente Alto & 4.555 & 50,2 \\
& La Pintana & 618 & 6,8 \\
& La Granja & 2.288 & 25,2 \\
& San Ramón & 518 & 5,7 \\
& Pirque & 845 & 9,3 \\
\multirow{2}{*}{ Zona } & San José de Maipo & 252 & 2,8 \\
& Urbano & 7.979 & 87,9 \\
\multirow{2}{*}{ Edad } & Rural & 1.097 & 12,1 \\
& 18-44 & 1.005 & 11,1 \\
& 45-64 & 4.398 & 48,5 \\
& 65 y más & 3.636 & 40,1 \\
\hline
\end{tabular}

Fuente: Elaboración propia en base a información del SSMSO, enero de 2014 a junio de 2016. 
Tabla 3. Cobertura de retinografía en los diabéticos del programa cardiovascular de la APS del SSMSO

\begin{tabular}{|lcccc|}
\hline Comunas & $\begin{array}{c}\text { n personas con } \\
\text { retinografía }\end{array}$ & $\begin{array}{c}\text { Total diabéticos } \\
\text { del PCV }\end{array}$ & $\begin{array}{c}\text { Cobertura de retinografía } \\
\text { en diabéticos del PSCV (\%) }\end{array}$ & $\begin{array}{c}\text { IC 95\% } \\
\text { Superior-Inferior }\end{array}$ \\
\hline Puente Alto & 4.618 & 23.057 & 20,03 & $19,46-20,61$ \\
La Pintana & 622 & 9.440 & 6,59 & $6,08-7,13$ \\
\hline La Granja & 2.351 & 5.164 & 45,53 & $43,7-47,4$ \\
\hline San Ramón & 523 & 4.757 & 10,99 & $10,07-11,98$ \\
\hline Pirque & 854 & 1.055 & 80,95 & $75,61-86,56$ \\
\hline San José de Maipo & 254 & 633 & 40,13 & $36,23-44,02$ \\
\hline Total & 9.222 & 44.106 & 20,91 & $20,53-21,29$ \\
\hline
\end{tabular}

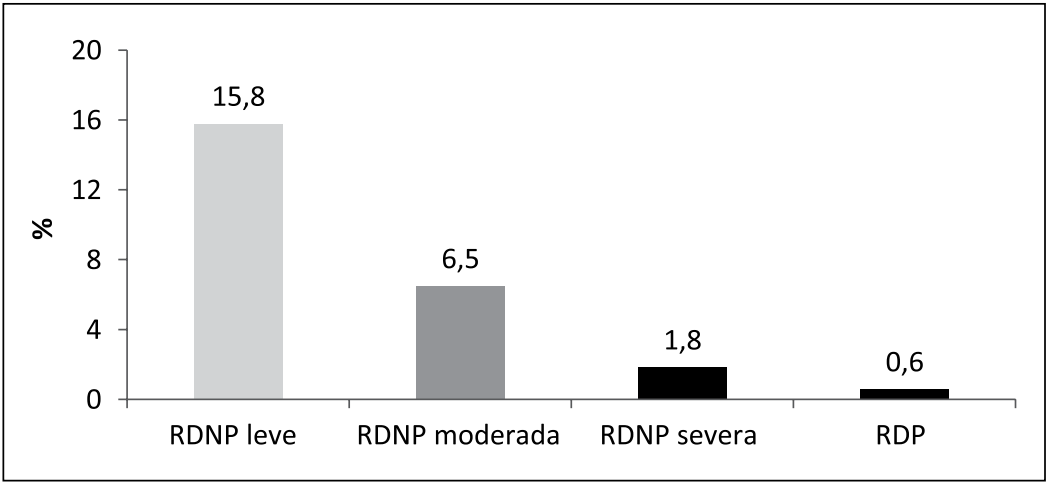

Figura 1. Severidad de retinopatía diabética (se excluye del gráfico el $75,4 \%$ que no tiene retinopatía diabética). RDNP: Retinopatía Diabética No Proliferativa. RDP: Retinopatía Diabética Proliferativa.
En relación a la cobertura, el tamizaje de retinografía en los diabéticos adscritos al PSCV es de 209 por 1.000 pacientes (Tabla 3). La mayor cobertura se observa en comunas en condición de ruralidad (Pirque y San José de Maipo). Entre las comunas urbanas destacan La Granja y La Pintana, con la mayor y menor cobertura, respectivamente.

La prevalencia de RD en los pacientes adheridos al PSCV es de 24,6 por cada 100 diabéticos, destacando según severidad, la RDNP leve con $15,8 \%$, alcanzando la RDNP severa y RDP un 2,4 por 100 (Figura 1). Asociado a cualquier clasificación de $\mathrm{RD}, 64$ pacientes $(0,7 \%)$ presentaron sospecha de EMD, ya que el diagnóstico propiamente tal sólo puede ser realizado por oftalmólogo.

$\mathrm{La}$ asociación de la $\mathrm{RD}$ según variables demográficas muestra que no hay diferencias significativas según sexo. Las comunas con mayor RDNP Severa y RDP corresponden a las de Puente Alto y La Pintana (3,2\% y 3,3\% respectivamente). Estas diferencias son estadísticamente significativas respecto de otras comunas del área geográfica $(\mathrm{p}<0,001)$. Cabe destacar que, en las comunas urbanas, la proporción de pacientes con RDNP Severa y RDP es $32,4 \%$ mayor que en las rurales (2,5\% urbano y $1,7 \%$ rural). La asociación según tramo de edad muestra que la RDNP severa y RDP en personas entre 18 y 44 años es $46 \%$ más, que en los mayores de 45 (3,9;2,2 respectivamente) (Tabla 4).

\section{Discusión}

La prevalencia de RD en nuestro estudio fue de $24,6 \%$, la cual concuerda con la reportada en la literatura internacional, que reporta prevalencias de $\mathrm{RD}$ entre $12,1 \%$ y $40,3 \%$ en pacientes diabéticos tipo $2^{5,14}$ y similar a las publicadas en los estudios de Verdaguer y Abuabuad, quienes reportan $28,5 \%$ y $24,8 \%$ respectivamente ${ }^{7,8}$. No obstante, resulta importante mencionar las diferencias 
Tabla 4. Prevalencia de retinopatía diabética según severidad y variables demográficas

\begin{tabular}{|c|c|c|c|c|c|c|c|}
\hline & & $\begin{array}{c}\text { RDNP } \\
\text { leve (\%) }\end{array}$ & $\begin{array}{c}\text { RDNP } \\
\text { moderada } \\
(\%)\end{array}$ & $\begin{array}{l}\text { RDNP } \\
\text { severa }\end{array}$ & RDP & $\begin{array}{l}\text { Sin retinopatía } \\
\text { diabética }\end{array}$ & $\begin{array}{c}\text { Valor } \\
\text { p }\end{array}$ \\
\hline Total & & $\begin{array}{c}15,8 \\
{[15,00-16,55]}\end{array}$ & $\begin{array}{c}6,5 \\
{[5,94-7,00]}\end{array}$ & $\begin{array}{c}1,8 \\
{[1,52-2,11]}\end{array}$ & $\begin{array}{c}0,6 \\
{[0,40-0,73]}\end{array}$ & $\begin{array}{c}75,4 \\
{[74,44-76,30]}\end{array}$ & \\
\hline Sexo & $\begin{array}{l}\text { Hombre } \\
\text { Mujer }\end{array}$ & $\begin{array}{c}16,2 \\
{[14,93-17,48]} \\
15,5 \\
{[14,50-16,50]}\end{array}$ & $\begin{array}{c}5,7 \\
{[4,94-6,50]} \\
6,9 \\
{[6,23-7,63]}\end{array}$ & $\begin{array}{c}2,0 \\
{[1,56-2,56]} \\
1,7 \\
{[1,33-2,05]}\end{array}$ & $\begin{array}{c}, 5 \\
{[0,25-0,80]} \\
.6 \\
{[0,38-0,81]}\end{array}$ & $\begin{array}{c}75,5 \\
{[74,02-77,00]} \\
75,3 \\
{[74,10-76,45]}\end{array}$ & 0,174 \\
\hline Comuna & $\begin{array}{l}\text { Puente Alto } \\
\text { La Pintana } \\
\text { La Granja } \\
\text { San Ramón } \\
\text { Pirque } \\
\text { San José de } \\
\text { Maipo }\end{array}$ & $\begin{array}{c}15,0 \\
{[13,91-16,08]} \\
14,8 \\
{[11,80-17,74]} \\
15,9 \\
{[14,30-17,44]} \\
18,2 \\
{[14,60-21,72]} \\
18,6 \\
{[15,80-21,39]} \\
17,2 \\
{[12,11-22,22]}\end{array}$ & $\begin{array}{c}7,6 \\
{[6,77-8,37]} \\
8,8 \\
{[6,40-11,14]} \\
3,8 \\
{[2,94-4,60]} \\
6,7 \\
{[4,34-9,02]} \\
6,3 \\
{[4,51-8,04]} \\
6,0 \\
{[2,74-9,27]}\end{array}$ & $\begin{array}{c}2,3 \\
{[1,87-2,80]} \\
3,1 \\
{[1,60-4,59]} \\
6 \\
{[0,26-1,00]} \\
1,7 \\
{[0,41-2,92]} \\
1,7 \\
{[0,70-2,73]} \\
1,3 \\
{[0,26-3,71]}\end{array}$ & $\begin{array}{c}, 8 \\
{[1,54-1,11]} \\
2 \\
{[0,00-0,95]} \\
5 \\
{[0,15-0,77]} \\
2 \\
{[0,00-1,15]} \\
, 1 \\
{[0,00-0,71]} \\
0,0 \\
{[0,00-1,57]}\end{array}$ & $\begin{array}{c}74,3 \\
{[72,95-75,60]} \\
73,2 \\
{[69,51-76,88]} \\
79,3 \\
{[75,54-81,02]} \\
73,3 \\
{[69,21-77,34]} \\
73,3 \\
{[70,16-76,50]} \\
75,5 \\
{[69,80-81,27]}\end{array}$ & 0,001 \\
\hline Zona & $\begin{array}{l}\text { Urbano } \\
\text { Rural }\end{array}$ & $\begin{array}{c}15,4 \\
{[14,60-16,26]} \\
18,3 \\
{[15,83-20,70]}\end{array}$ & $\begin{array}{c}6,5 \\
{[5,94-7,07]} \\
6,2 \\
{[4,68-7,75]}\end{array}$ & $\begin{array}{c}1,9 \\
{[1,53-2,16]} \\
1,6 \\
{[0,76-2,40]}\end{array}$ & $\begin{array}{c}, 6 \\
{[0,44-0,81]} \\
, 1 \\
{[0,00-0,55]}\end{array}$ & $\begin{array}{c}75,6 \\
{[74,60-76,55} \\
73,8 \\
{[71,08-76,60]}\end{array}$ & 0,044 \\
\hline $\begin{array}{l}\text { Tramos } \\
\text { de edad }\end{array}$ & $\begin{array}{l}\text { 18-44 } \\
\text { 45-64 } \\
65 \text { y más }\end{array}$ & $\begin{array}{c}15,5 \\
{[13,17-17,90]} \\
15,3 \\
{[14,21-16,45]} \\
16,4 \\
{[15,15-17,67]}\end{array}$ & $\begin{array}{c}9,3 \\
{[7,40-11,20]} \\
5,8 \\
{[5,03-6,48]} \\
6,5 \\
{[5,70-7,37]}\end{array}$ & $\begin{array}{c}3,2 \\
{[2,00-4,34]} \\
1,8 \\
{[1,37-2,20]} \\
1,5 \\
{[1,05-1,89]}\end{array}$ & $\begin{array}{c}, 7 \\
{[0,14-1,34]} \\
3 \\
{[0,13-0,50]} \\
, 8 \\
{[0,50-1,14]}\end{array}$ & $\begin{array}{c}71,2 \\
{[68,31-74,18]} \\
76,8 \\
{[75,50-78,11]} \\
74,8 \\
{[73,30-76,24]}\end{array}$ & 0,001 \\
\hline
\end{tabular}

Fuente: Elaboración propia en base a información del SSMSO, enero de 2014 a junio de 2016. RDNP: Retinopatía Diabética No proliferativa. RDP: Retinopatía Diabética Proliferativa.

metodológicas entre los estudios. En primer lugar, los pacientes evaluados en el estudio de Verdaguer fueron en el contexto de una campaña ("El Día D"). Por lo tanto, es esperable que fuesen pacientes de mayor riesgo para presentar $\mathrm{RD}$, existiendo un posible sesgo de selección al concurrir personas con mayor interés, mientras que los pacientes del estudio de Abuabuad fueron evaluados en el contexto de un estudio aislado en un centro APS, donde no se reportan coberturas. Por otra parte, el total de personas evaluadas en los dos estudios mencionados alcanzan un total de 1.816 , mientras que el presente análisis expone los resultados de 9.076 pacientes de un programa de tamizaje oficial de RD. Otra diferencia metodológica a destacar es que, en nuestra investigación, la evaluación y clasificación del grado de $\mathrm{RD}$ es realizada por el TM, quien deriva al servicio de oftalmología del CARS en caso de sospechar RD. Experiencias internacionales avalan los métodos de tamizaje mediante retinografías digitales, como también las evaluaciones de éstas por TM debidamente capacitados ${ }^{20,24,25}$. En el Reino Unido existe un programa nacional de tamizaje de $\mathrm{RD}$ que reporta los mejores resultados en la literatura ${ }^{26}$, en el cual la clasificación de las retinografías es realizada por tecnólogo u otro personal no médico, capacitado y acreditado. A su vez el programa es sometido a constantes evaluaciones de calidad, presentando reportes anuales de resultados ${ }^{16,20}$. El programa 
de tamizaje de RD del SSMSO tiene pendiente aún los estudios de validez y las evaluaciones de calidad, los cuales permiten una mejor validación del modelo.

Por otra parte, en nuestro estudio $61,5 \%$ de los pacientes evaluados fueron mujeres, lo cual es coincidente con los dos reportes mencionados anteriormente, que muestran $61,6 \%$ y $60,9 \%$ respectivamente. Verdaguer hipotetiza una mayor responsabilidad por parte de las mujeres, aseveración que sin duda llama la atención y que necesita mayor profundización, ya que otra hipótesis plausible es que podría existir un porcentaje importante de hombres que no se está realizando el tamizaje de RD o, que la incidencia de la complicación sea de diferente magnitud según sexo.

Los resultados muestran también que aquellos pacientes de las comunas consideradas urbanas presentaron con mayor frecuencia hallazgos compatibles con $\mathrm{RD}$ ( 1,32 veces), comparado con aquellos de comunas rurales (cualquiera fuese su severidad), resultado que no tiene una clara explicación. Por ello resulta necesario realizar estudios que permitan profundizar en estos resultados.

Llama la atención que, en aquellos pacientes con edades entre 18 y 44 años, se pesquisó $46 \%$ más de $\mathrm{RD}$ en comparación con los de mayor edad ( $\geq 45$ años). Estos resultados permiten hipotetizar que probablemente los pacientes jóvenes con DM tipo 1 tienen un mal control metabólico, y aquellos con DM tipo 2 ya presentan daños secundarios (como la RD) al momento del diagnóstico. No es descartable un sesgo de selección por la natural atrición dada por la mayor mortalidad de pacientes diabéticos de mayor edad. Adicionalmente, en nuestros hallazgos destaca un bajo porcentaje de sospecha de EMD: se trata sólo de 64 pacientes $(0,7 \%)$. En series internacionales la prevalencia del EMD alcanza hasta 7\%. Verdaguer y Abuabuad describieron $5 \%$ y $3,8 \%$ respectivamente en pacientes chilenos ${ }^{7,8}$. Esta diferencia se podría explicar por el diseño del protocolo de tamizaje, cuyo objetivo inicial no fue considerar la pesquisa de EMD. Este antecedente es preocupante ya que el EMD es la principal causa de disminución de la agudeza visual en los pacientes diabéticos y cuyo tratamiento debe ser instaurado precozmente, para así lograr tener buenos resultados visuales ${ }^{27,28}$.

La RD es un patología que cumple con los criterios de la OMS para beneficiarse del tamizaje $\mathrm{e}^{10-12}$. Su correcta implementación ha demostrado su efectividad en la prevención de la ceguera en los pacientes diabéticos, y presenta beneficios costo económico ampliamente demostrado en la literatura $^{13}$. El año 2000 Steffason et al, concluyó que, desde el punto de vista de salud pública, el tamizaje de la RD es uno de los procedimientos médicos de mejor costo-efectividad ${ }^{29}$. En el Reino Unido, los programas de tamizaje han logrado coberturas que superan $80 \%$ y los resultados han sido alentadores ${ }^{16}$. Liew et al, publicó una comparación de las causas de ceguera en adultos trabajadores, pre y post implantación del programa de tamizaje, donde la retinopatía deja de ser la primera causa de ceguera, dando paso a los trastornos hereditarios de la retina, como patología poco frecuente ${ }^{30}$.

En relación a la cobertura del tamizaje nacional, como uno de los objetivos del presente análisis, los resultados son poco alentadores, considerando una cobertura de sólo 21\% de los inscritos en el PSCV del SSMSO. Desde junio de 2006, la RD está considerada una patología GES (Ley 19.966), programa que asegura la realización de tamizaje de RD anual. Sin embargo, no hay datos informados respecto a dicha cobertura en la literatura nacional, siendo éstos los primeros resultados en publicarse $\mathrm{e}^{17,18}$.

El presente estudio aporta conocimiento inédito sobre cobertura de tamizaje de $\mathrm{RD}$ a nivel nacional, entregando un primer diagnóstico acerca de lo que se está realizando rutinariamente. Es esencial trabajar para el desarrollo de programas de tamizajes oficiales, que realicen la publicación sistemática de sus resultados, permitiendo el análisis y una mejora continua de los mismos. Así mismo, resulta importante la realización de estudios en Chile que validen los distintos modelos de tamizajes y la participación de TM en el proceso. Es conveniente aumentar potencialmente las coberturas, especialmente en los estratos socioeconómicos más bajos que muestran una mayor prevalencia de detección de RD y menores tasas de cobertura en los programas de tamizaje $e^{31,32}$.

Agradecimientos: Se agradece la cooperación de la Dra. Luz María Durango Henao, de la unidad de estudios de la subdirección de gestión asistencial del SSMSO por facilitar las bases de datos usadas para el desarrollo de este estudio; a Ana María Jasmen, bibliotecóloga de la Universidad del Desarrollo, por ayudarnos en la búsqueda de material bibliográfico necesario para este docu- 
mento, y al Dr. Carlos Cruz Marín y al Sr. Lucas Costa Ayala por la revisión del manuscrito.

\section{Referencias}

1. Furtado J, Lansingh V, Carter M, Milanese M, Peña B, Ghersi $\mathrm{H}$, et al. Causes of blindness and visual impairment in Latin America. Surv Ophtalmol 2012; 57 (2): 149-77.

2. Wild S, Rogloc G, Green A, Sicree R, King H. Global prevalence of diabetes: estimates for the year 2000 and projections for 2030. Diabetes Care 2004; 27: 1047-53.

3. Chile. Ministerio de Salud. Resultados Encuesta Nacional de Salud, Chile 2009-2010. Santiago: MINSAL, disponible en: http://www.redsalud.gov.cl/portal/url/ item/99bbf09a908d3eb8e04001011f014b49.pdf [consultado el 23 de julio de 2016).

4. Chile. Ministerio de Salud. Evaluación de las características y cambios en el tratamiento de la diabetes en la población general chilena. ENS 2003 y ENS 2009-10. Santiago: MINSAL, disponible en: http://web.minsal.cl/ sites/default/files/files/Informecoberturaefectivadiabetes. pdf [consultado el 23 de diciembre de 2016].

5. Klein R, Klein B, Moss S, Cruickshanks K. The Wisconsin Epidemiologic Study of Diabetic Retinopathy: XVII. The 14-year incidence and progression of diabetic retinopathy and associated risk factors in type 1 diabetes. Ophthalmology 1998; 105: 1801-15.

6. Yau J, Rogers S, Kawasaki R, Lamoureux E, Kowalski J, Bek T, et al. Global prevalence and major risk factors of diabetic retinopathy. Diabetes Care 2012; 35 (3): 556-64.

7. Barría F, Martínez F. Guía práctica clínica de retinopatía diabética para Latinoamérica, 2012. Disponible en: http://www.icoph.org/dynamic/attachments/taskforce_documents/panamericandiabeticretinopathyguide2011-spanish.pdf [consultado el 11 de agosto de 2016].

8. Verdaguer J, Vicencio C, Zúñiga C, Molina, E. Tamizaje para Retinopatía Diabética en Latinoamérica (Día D). Resultados. Arch. Chil. Oftal 2001; 58: 39-43.

9. Abuabuad S, Guzmán P, Urzúa C. Prevalencia de Retinopatía Diabética y Edema macular en población Diabética del CESFAM Cordillera Andina de los Andes. Revista Chilena de Salud pública 2012; 18 (1): 81-6.

10. Echouffo-Tcheugui J, Ali M, Roglic G, Hayward R, Narayan K. Screening intervals for diabetic retinopathy and incidence of visual loss: a systematic review. Diabet Med 2013; 30: 1272-92.

11. Harding S, Broadbent D, Neoh C, White M, Vora J. Sensitivity and specificity of photography and direct ophthalmoscopy in screening for sight threatening eye disease: the Liverpool Diabetic Eye Study. BMJ 1995; 301 (7013): 1131-5.

12. Pieczynsky J, Grzyybowski A. Review of Diabetic Retinopathy Screening Methods and Programmes Adopted in Different Parts of the World. European Ophthalmic Review 2015; 9 (1): 49-55.

13. Dasbach E, Fryback D, Newcomb P, Klein R, Klein BE. Cost-effectiveness of strategies for detecting diabetic retinopathy. Med Care 1991; 29 (1): 20-39.

14. Squirrel D, Talbot J. Screening for diabetic retinopathy. J R Soc Med 2003; 96 (6): 273-6.

15. Canadá. The association of Faculties of Medicine of Canada. Disponible en: http://phprimer.afmc.ca/ Part3-PracticeImprovingHealth/Chapter9Screening/ Criteriaforintroducingascreeningtest [consultado el 23 de diciembre de 2016].

16. Scanlon $\mathrm{P}$. The English national screening programme for sight-threatening diabetic retinopathy. J Med Screen 2008; 15: 1-4.

17. Chile. Ministerio de Salud. Guía Clínica de Retinopatía Diabética, Chile 2010. 2 Ed. Santiago: MINSAL.

18. Escobar M, Obreque A. Implementación del enfoque de riesgo en el programa de salud cardiovascular. 2002. Disponible en: http://pifrecv.utalca.cl/docs/2009/Implementacion_Enfoque_Riesgo_PSCV_29_julio.pdf [consultado el 3 de septiembre de 2016].

19. Chile. Servicio de Salud Metropolitano Sur Oriente. Memorias Complejo Asistencial Dr. Sotero del Río, Chile 2014. Santiago: SSMSO. Disponible en: http:// www.hospitalsoterodelrio.cl/home/files/memoriacasr/ memoria/memoriacasr2014.pdf [consultado el 19 de julio de 2016].

20. Olson J, Strachan F, Hipwell J, Goatman K, McHardy $\mathrm{K}$, Forrester J, et al. A comparative evaluation of digital imaging, retinal photography and optometrist examination in screening for diabetic retinopathy. Diabetic Medicine 2003; 20 (7): 528-34.

21. Sharp P, Olson J, Strachan F, Hipwell J, Ludbrook A, O'Donnell M, et al. The value of digital imaging in diabetic retinopathy. Health Technology Assessment 2003; 7 (30): 1-119.

22. Scanlon P, Malhotra R, Thomas G, Foy C, Kirkpatrick J, Lewis-Barned N, et al. The effectiveness of screening for diabetic retinopathy by digital imaging photography and technician ophthalmoscopy. Diabetic Medicine 2003; 20 (6): 467-74.

23. Wilkinson C, Ferris III F, Klein R, Lee P, Agardh C, Davis $\mathrm{M}$, et al. Proposed International Clinical Diabetic Macular Edema Disease Severity Scales. Ophthalmology 2003; 1: 1677-82. 
24. Bhargava M, Cheung C, Sabanayagam C, Kawasaki R, Harper C, Lamoureux E, et al. Accuracy of diabetic retinopathy screening by trained non-physician graders using non-mydriatic fundus camera. Singapore Med J 2012; 53: 715-9.

25. Schütt F, Bruckner T, Schäfer K, Lehnhoff D, Rudofsky $\mathrm{G}$, Kasperk C, et al. Fundus screening by medical technicians. Ophthalmology 2013; 110 (2): 154-9.

26. Harris M. The NHS Diabetic Eye Screening Programme: New Common Pathway. The Royal College of Ophthalmologists. London: Focus; 2012.

27. Williams R, Airey M, Baxter H, Forrester J, Kennedy-Martin T, Girach A. Epidemiology of diabetic retinopathy and macular oedema: a systematic review. Eye. 2004; 18 (10): 963-83.

28. Wells J, Glassman A, Ayala A, Jampol L, Aiello L, Antoszyk A, et al. Aflibercept, bevacizumab, or ranibizu- mab for diabetic macular edema. N Engl J Med 2015; 372 (13): 1193-203.

29. Stefansson E, Bek T, Porta M, Larsen N, Kristinsson JK, Agardh E. Screening and prevention of diabetic blindness. Acta Ophthalmologica Scandinavica 2000; 78 (4): 374-85.

30. Liew G, Michaelides M, Bunce C. A comparison of the causes of blindness Certifications in England and Wales in working age adults (16-64 years), 1999-2000 with 2009-2010. BMJ Open 2014; 4: e004015.

31. Scanlon P, Carter S, Foy C, Husband R, Abbas J, Bachmann M. Diabetic retinopathy and socioeconomic deprivation in Gloucestershire. Journal of Medical Screening 2008; 15 (3): 118-21.

32. Leese G, Boyle P, Feng Z, Emslie-Smith A, Ellis J. Screening uptake in a well-established diabetic retinopathy screening program: the role of geographical access and deprivation. Diabetes Care 2008; 31 (11): 2131-5. 